\title{
Editorial \\ Foodomics, foodome and modern food analysis
}

Since the first definition of Foodomics in 2009 (asa discipline that studies the food and nutrition domains through the application and integration of advanced omics technologies to improve consumer's well-being, health, and confidence), many works have already shownthe tremendous possibilities of this approach to boost food science investigations, including those related to speed up the resolution of food safety issues, to improve food quality and food traceability, and to understand at molecular level the bioactivity of food and food ingredients in our body. To help solving these important issues, the concept of Foodomeis now defined as the collection of all compounds present in any investigated food sample and/or in any biological system interacting with the investigated food at a given time. Of course, many challenges still remain unresolved, while new needs and demands are continuously coming out in this area of research, which gives an idea of the complexity of this discipline. For instance, in a recent work published by M. Dehghan et al., in The Lancet (29th August 2017, DOI:http://dx.doi.org/10.1016/S0140-6736(17)32252-3) it is highlighted the controversial relationship between macronutrients and cardiovascular disease and mortality. Thus, in a cohort study of the dietary intake of 135335 individuals with a median follow up of 7.4 years, it was concluded that high carbohydrate intake was associated with higher risk of total mortality, whereas total fat and individual types of fat were related to lower total mortality. Moreover, total fat and types off at were not associated with cardiovascular disease, myocardial infarction, or cardiovascular disease mortality, whereas saturated fat had an inverse association with stroke. In summary, the authors conclude that "global dietary guidelines should be reconsidered inlight of thesefindings". This paper is a good example of our poor knowledge on many basic aspects related to the long-term effectof foods and ingredients on our health, which also highlights the long way to go till successfully achieving the so called personalized nutrition (welcome to Far Far Away). This special issue on Foodomics is a good illustration of the tolos that can be used to help overcoming some challenges and new demands on different topics related to Food Science and Nutrition. Thus, it is discussed how targeted and non-targeted approaches using omics technologies, including transcriptomics, proteomics and metabolomics, can provide new insights about the molecular mechanism that can explain the bioactivity/safety/quality of food and food compounds leading to the discovery of new biomarkers. Logically, the developments in this modern discipline go parallel to the growth of other advanced analytical methodologies, whichare generally used in fundamental food studies regarding foodsafety, authenticity and quality. As an overall conclusion, it can be said that nowadays Foodomics and advanced food analysis are attracting more and more attention from research institutions, agencies, food industries, regulatory laboratories and scientific instrumentation manufacturers as demanded by government food regulations and consumers. The interest in Foodomics and innovative food analysis and the different trends in this hot area of research are well documented in the present special TrAC issue. Namely, this issue on "Foodomics and Modern Food Analysis" presents 17 review papers dedicated to: i) Foodomics evaluation of bioactive compounds in foods; ii) Foodomics in food microbiology; iii) Foodomics to investigate the mycobolome; iv) Green Foodomics; v) Recent chemometrics advances for Foodomics; vi) Precision food safety by genomics tools; vii) Mass spectrometry-based metabolomics approaches for food safety, quality and traceability; viii) Metabolomics to evaluate the impact of nutritional and physiological challenges; ix) Non-targeted screening of chemical contaminants and illegal additives in food; $x$ ) Novel mass spectrometry approaches in food proteomics; xi) Omics methods to investigate food allergies; xii) Multidimensional liquid chromatography in food analysis; xiii) Multidimensional gas chromatography in food analysis; xiv) Advanced analysis of polysaccharides, novel functional components in food and medicine dual purposes Chinese herbs; 
xv) Chiralseparations in food analysis; xvi) Recent applications of nanomate-rials in food safety and xvii) Recent developments and applications of mass spectrometry for the quality and safety assessment of cooking oil. As Editor of this special TrAC issue devoted to "Foodomics and Modern Food Analysis", I would like to thank all the authors for their suitable contributions, all reviewers for the time they devoted to the evaluation of the papers, and to those of TrAC team (especially Prof. Janusz Pawliszyn, Ms. Angela Yuan and Ms. Dawn Mahoney) who contributed with their support and effort for thepreparation of this special issue.

Muchas gracias a todos!

Alejandro Cifuentes

Laboratory of Foodomics, CIAL, CSIC, Madrid, Spain

E-mail address:a.cifuentes@csic.es. 